\title{
Cardiopulmonary Exercise Testing in Systemic Sclerosis: 'Ars longa, vita brevis'
}

\author{
Vasilios Tzilas Demosthenes Bouros \\ First Academic Department of Pneumonology, Medical School, National and Kapodistrian University of Athens, and \\ Hospital for Diseases of the CHEST 'SOTIRIA', Athens, Greece
}

True knowledge exists in knowing that you know nothing. Socrates

Systemic sclerosis (SSc) is a collagen tissue disease that can affect a variety of organs. Its pathophysiology includes aberrant fibroblast proliferation resulting in excessive production and deposition of collagen leading to fibrosis, endothelial dysfunction resulting in small vessel vasculopathy and immunological abnormalities $[1,2]$. Thus, SSc can affect multiple organs in multiple ways. Since the effective management of scleroderma renal crisis with the use of angiotensin-converting enzyme inhibitors, cardiopulmonary involvement has become the major cause of morbidity and mortality [3]. Cardiopulmonary involvement itself is quite diverse as it encompasses interstitial lung disease, mainly of non-specific interstitial pneumonia histology [4], pulmonary hypertension and cardiac involvement, all of which can present as dyspnea and exercise limitation. In some cases, determining the cause of symptoms is straightforward, for example in extensive interstitial disease with a restrictive functional pattern and a normal heart echo or in cases with minimal fibrosis, symptoms and signs of right heart failure and an elevated systolic pulmonary artery pressure (sPAP) on Doppler echocardiography. However, this is not always

\section{KARGER}

E-Mail karger@karger.com

www.karger.com/res the case, and there is also the possibility of co-existent pathophysiologic mechanisms. In the latter case, it is important to identify the primary cause of the symptoms. Another important parameter is the ability to recognize organ and type of involvement at an early stage in the course of the disease that will enable early intervention.

In the latest issue of Respiration, Boutou et al. [5] demonstrated that maximal cardiopulmonary exercise testing (CPET) on a cyclic ergometer can provide a useful tool in assessing the main cause of exercise limitation. Their study included a large number of clinically stable SSc patients (both diffuse and limited) who presented due to perceived exertional dyspnea or decreased physical performance and were referred for functional evaluation. The vast majority of patients were able to successfully complete CPET (78 out of $82,95 \%$ ). The reasons for not successfully completing CPET were discoordination with the bike ( 1 patient) and joint pain and stiffness ( 3 patients). Thus, performing CPET is feasible in everyday clinical practice. Using a modified algorithm [6], it was possible to categorize the patients into 4 distinct subgroups: (a) normal exercise capacity or subnormal but not limited by evident heart or lung disease $(\mathrm{N}),(\mathrm{b})$ respiratory limitation (RL), (c) left ventricular dysfunction (LVD), and (d) pulmonary vasculopathy (PV) and define
(C) 2016 S. Karger AG Basel

0025-7931/16/0913-0202\$39.50/0
Prof. Demosthenes Bouros, MD, PhD, FERS, FCCP, FAPSR

First Academic Department of Pneumonology, Medical School University of Athens, Hospital for Diseases of the CHEST 'SOTIRIA' Messogion Ave. 152, GR-11527 Athens (Greece)

E-Mail dbouros@med.uoa.gr 
the main cause of exercise limitation. The majority of patients $(32.1 \%)$ presented with PV, $25.6 \%$ presented with LVD, $10.2 \%$ with RL, while $32.1 \%$ had normal/subnormal exercise capacity. It is interesting that respiratory limitation was the cause of exercise limitation and dyspnea in the minority of patients.

As expected, the extent of fibrosis on high-resolution computed tomography favored the presence of RL. However, we must note that $40 \%$ of LVD patients also presented with extensive fibrosis. Also, the majority of LVD patients were positive to anti-Scl 70. From a pathogenetic standpoint, this is in line with the hallmark cardiac manifestation of SSc, which is myocardial fibrosis that can remain subclinical for a long period [7]. From a clinical view, it highlights the possibility that besides obvious pulmonary involvement, heart dysfunction can contribute importantly to the patient's symptoms and warrant specific evaluation and treatment. In this context, CPET can provide clinically important information. Of note, the LVD group presented the worst exercise capacity.

The PV group was difficult to differentiate from the other groups. $\mathrm{PETCO}_{2}$ at peak $\mathrm{VO}_{2}$ could discriminate $\mathrm{PV}$ from either the $\mathrm{N}$ or RL but not from the LVD group. The differentiation from the latter group was based on a decreasing (contrasting to a neutral or increasing) $\mathrm{PETCO}_{2}$ from rest to anaerobic threshold. It is noteworthy that sPAP (assessed by Doppler echocardiography) was not a discriminatory parameter between the PV and LVD group. Furthermore, some patients in the PV group had sPAP $<35 \mathrm{~mm} \mathrm{Hg}$, and the peak work rate \% predicted was not different between the PV and the $\mathrm{N}$ group. Hence, (i) it is difficult to diagnose the PV group based solely on clinical parameters, and (ii) it is possible to non-invasively diagnose pulmonary vasculature involvement early in the course of disease by CPET. This is in line with the findings by Dumitrescu et al. [6].

The retrospective nature of the study is an obvious inherent limitation. Also, data from right heart catheterization, indeed difficult to acquire, nevertheless would increase the validity of the study. Furthermore, conducting subgroup analysis of the exercise limitation phenotypes based on the presence of diffuse versus limited scleroderma would be clinically useful. Regarding the LVD subgroup, we would like to have more data on the type of cardiac involvement based on echocardiography and their correlation with CPET parameters. Finally, it is surprising that DLCO was not statistically different between the PV and $\mathrm{N}$ groups.

There is an unmet need for early detection of pulmonary arterial hypertension in SSc before hemodynamic deterioration and clinically overt disease. This is extremely important given the fact that pulmonary hypertension in the context of SSc carries a more ominous prognosis comparing to idiopathic pulmonary hypertension [8]. Indeed, screening for pulmonary arterial hypertension by Doppler echocardiography in patients with SSc resulted in earlier diagnosis and improved survival [9]. The opportunity of defining pulmonary vasculature involvement by CPET early in the course of the disease not only creates hope but also raises several questions. We need further prospective studies to establish the precise progression of the PV group in the course of time and eventually investigate if early therapeutic intervention can delay the progression of pulmonary hypertension and improve patients' outcomes. Obtaining prospectively further data about the clinical role and natural history of the aforementioned phenotypes in SSc will allow us to better define the role and value of CPET in clinical practice.

\section{References}

$>1$ Fischer A, du Bois R: Interstitial lung disease in connective tissue disorders. Lancet 2012; 380:689-698.

$\checkmark 2$ Papiris SA, Manali ED, Kolilekas L, et al: Investigation of lung Involvement in connective tissue disorders. Respiration 2015;90:2-24.

$\checkmark 3$ Elhai M, Meune C, Avouac J, et al: Trends in mortality in patients with systemic sclerosis over 40 years: a systematic review and metaanalysis of cohort studies. Rheumatology (Oxford) 2012;51:1017-1026.

4 Bouros D, Wells AU, Nicholson AG, et al: Histopathologic subsets of fibrosing alveolitis in patients with systemic sclerosis and their

Cardiopulmonary Exercise Testing in

Systemic Sclerosis relationship to outcome. Am J Respir Crit Care Med 2002;165:1581-1586.

5 Boutou AK, Pitsiou GG, Siakka P, Dimitroulas T, Paspala A, Sourla E, Chavouzis N, Garyfallos A, Argyropoulou P, Stanopoulos I: Phenotyping exercise limitation in systemic sclerosis: the use of cardiopulmonary exercise testing. Respiration 2016;91:115123.

-6 Dumitrescu D, Oudiz RJ, Karpouzas G, et al: Developing pulmonary vasculopathy in systemic sclerosis, detected with non-invasive cardiopulmonary exercise testing. PLoS One 2010;5:e14293.
7 Kahan A, Coghlan G, McLaughlin V: Cardiac complications of systemic sclerosis. Rheumatology (Oxford) 2009;48(suppl 3):iii45-iii48.

$>8$ McLaughlin V, Humbert M, Coghlan G, et al: Pulmonary arterial hypertension: the most devastating vascular complication of systemic sclerosis. Rheumatology (Oxford) 2009; 48(suppl 3):iii25-iii31.

9 Humbert M, Yaici A, de Groote P, et al: Screening for pulmonary arterial hypertension in patients with systemic sclerosis: clinical characteristics at diagnosis and long-term survival. Arthritis Rheum 2011;63:35223530 . 